\title{
"Buoyancy" in granular Medium: How deep can an object sink in sand?
}

\author{
Yahya Sandali ${ }^{\mathrm{a}}$, Ram Chand ${ }^{\mathrm{b}, *}$, Qingfan $\mathrm{Shi}^{\mathrm{a}}$ \\ ${ }^{a}$ Department of Physics, Beijing Institute of Technology, Beijing 100081, China \\ ${ }^{b}$ Center for Theoretical and Computational Physics, \\ Department of Physics, Faculty of Science, \\ University of Malaya, Kuala Lumpur 50603, Malaysia
}

\begin{abstract}
The behavior of granular matter is different from either fluids or solids. One may not be able to answer even a naive question such as how deep an object can sink in sand. Answers to the depth of footprints on sand beach and its dependence on grain size have never been seriously studied before, and may deserve a closer look and better understanding. Laying a ball of fixed size onto granules, we have measured the sinking depth $(S D)$ of the ball into granules of different sizes and studied the dependence of $S D$ on the sizes of the ball and granules. We find that the $S D$ is very sensitive to the size of granules and the variation of $S D$ on granule size is not monotonic. The maximum $S D$ occurs at $r \approx \frac{1}{20} R$, where $r$ and $R$ are the radii of granules and the ball, respectively. This ratio does not depend on the density of the ball and the volume fraction of granules. An empirical formula of $S D$ on densities and sizes of the ball and granules are obtained based on the experimental results.
\end{abstract}

\footnotetext{
*Corresponding author

Email address: ram.chand2k11@yahoo.com (Ram Chand)
} 
Keywords: Granular buoyancy, sinking depth, sand

\section{Introduction}

Granular materials have recently become a popular subject of studies [1] 2] [3] because of their scientific and technological importance. Our understanding of properties of granular materials is far from complete, such as 5 jamming transition in static granular assemblies [4, a static assembly of noncohesive, spherical particles in contact [1] [5]. Particles within this system are under stress, supporting the weight of the material above them in addition to any applied load. It is known that stresses in a granular assembly are distributed in a highly inhomogeneous manner, along networks containing the largest inter-particle forces known as force chains [6] [7]. The assembly will behave like a solid when the force chains are strong enough to hold the assembly together and will flow like a fluid when these force chains are broken under stress [8]. Obviously, properties of these contact force chains crucially determine the bulk properties of the assembly, especially its load bearing 15 capability [9].

The formation of the force chains is the result of the inherently nonlinear nature of inter-particle friction forces and the particle's nearly hardsphere interaction [10][11]. The forces between granules are characterized by their contacts as they vanish identically when the granules are not in contact [12] [13] [3] [14. An important characteristic of the force chains is that their distribution in the granular assembly will be modified in response to an external stress[11][15]. When under stress, the particles start deforming. New contacts are created, and additional force chains are created. For that 
reason, a quantitative understanding of the force chains will be needed to understand the response of the granular assembly to an external stress. However, detailed studies of changes in these contacts and their corresponding forces are proved to be difficult because forces measurement inside a granular assembly is still not possible even if the stress pattern has been observed in a two-dimensional granular material under compression [16] [17].

30

One of the most important finding of these studies is that the force chains support most of the external load, effectively shielding large regions of the material [18]. Presumably, the characteristics of the force chains can be inferred from the external response of the granular assembly to a well-defined external stress without the need of measurements in the interior of the assembly if a reasonable model can be used[11] [19] [20] [22] [23] [24]. For example, when an intruder is lowered into a granular bed, properties of the contact force between the intruder and the bed will be related to the sinking depth of the intruder, which is the response of the bed [21][25]. In this letter, we report results of such an experiment. Properties of contact forces $F$ in granular beds with beads of size $r$ and density $\rho_{r}$ are probed by experiments in which the sinking distance $d$ of an intruder of size $R$ and density $\rho_{R}$ is measured as a function of $r$ when the intruder is lowered into the beds under its own weight. It is found that $d$ is not monotonic and its maximum sinking can be understood as the balance between the changes in $F$ and the number of contacts as $r$ is varied. The ratio $r / R$ and $\rho_{r} / \rho_{R}$ are found to be important parameters, and the contact forces are highly nonlinear in nature. 


\section{Experimental setup}

Our experimental setup is very simple. As is shown in the inset of Figure 1(a), a cylindrical container of an inner diameter $72 \mathrm{~mm}$ and a height $50 \mathrm{~mm}$ is filled with beads. Directly above the granular bed is an intruder ball, which is suspended from a vertical translation stage so that the position of the intruder can be controlled. The granular beds studied are all disordered three-dimensional, compact random packing of smooth glass beads (spheres) with 21 different radii in a range of $r=0.02 \mathrm{~mm}-1.8 \mathrm{~mm}$ with a size dis55 persion of about 20\%. The density of the glass beads is $\rho_{r}=2.5 \mathrm{~g} / \mathrm{cm}^{3}$. Eight radii of intruder balls (stainless steel balls used in ball bearing) are used: $1.50 \mathrm{~mm}, 2.55 \mathrm{~mm}, 3.15 \mathrm{~mm}, 4.00 \mathrm{~mm}, 5.00 \mathrm{~mm}, 5.75 \mathrm{~mm}, 7.50 \mathrm{~mm}$ and $10.30 \mathrm{~mm}$. Density of the intruder balls is $\rho_{R}=7.8 \mathrm{~g} / \mathrm{cm}^{3}$. In order to maintain a constant bulk packing density of the beds for various bead sizes, we always keep the volume of the granules and the total weight constant. The total mass in the experiment is kept a constant of $370 \mathrm{~g}$. The volume of the granular beds is then kept constant by various operations such as shaking, removal and addition of beads. In the experiments, the volume fraction is kept as $0.61 \pm 0.01$. During the compaction of the granular bed in the procedure above, the surface of the bed is always leveled off. The intruder ball is suspended from a fine cotton cord and lowered onto the surface of the granular packing as slowly as possible. The response of the beds to an intruder lowered into the beds under gravity is measured by the maximum distance that the intruder can travel after the intruder is in contact with the bed. This distance is defined as the $S D$ of the intruder. The initial (contact with the bed) and final positions of the intruder are measured by a traveling 
microscope. The measured $d$ will be a function of the dimension of the container for the granular bed if the container is not large enough to eliminate boundary effects. The container used in our experiment is tested to be large enough by comparing measured $d$ with a larger container. All measurements reported in this report are averaged over ten times to minimize statistical error. It should be pointed out that the intruder ball should be large and heavy enough when compared to the weight of the beads because there is a threshold value of $S D$ for one kind of bed (Fig. 3). Also the intruder should not be small to comparable with the size of the beads, otherwise big measurement-error will result.

\section{Results and discussion}

Figure 1(a) is the measured $S D$, shown as $d$, for an intruder in granular beds as a function of bead sizes $r$ with different intruder radius $R$. (Due to too many sets of data in the figure, the error bars are not shown. Error bars are shown in Figure 2.) When $r$ is small, $d$ increases with $r$. After reaching a maximum $d_{\max }, d$ begins to decrease at larger $r$. A remarkable feature of Figure 1(a) is that the measured $d$ is not a monotonic function of the bead sizes. There is a specific value of bead size $r *$ at which $d$ is maximum or the bed seems to be weakest.

When we re-plot the ratio of $d / R$ versus $r / R$ in Figure 1(b), the curves of different $R$ almost overlap. This indicates $d$ (at a fixed $r$ ) and $d_{\text {max }}$ increases linearly with $R$, and that $d_{\max }$ is found at a characteristic $\lambda=r / R \approx 0.05$. In other words, the sinking depth of an intruder reaches a maximum when its

size is about 20 times of the size of the beads. In our experimental condition, 

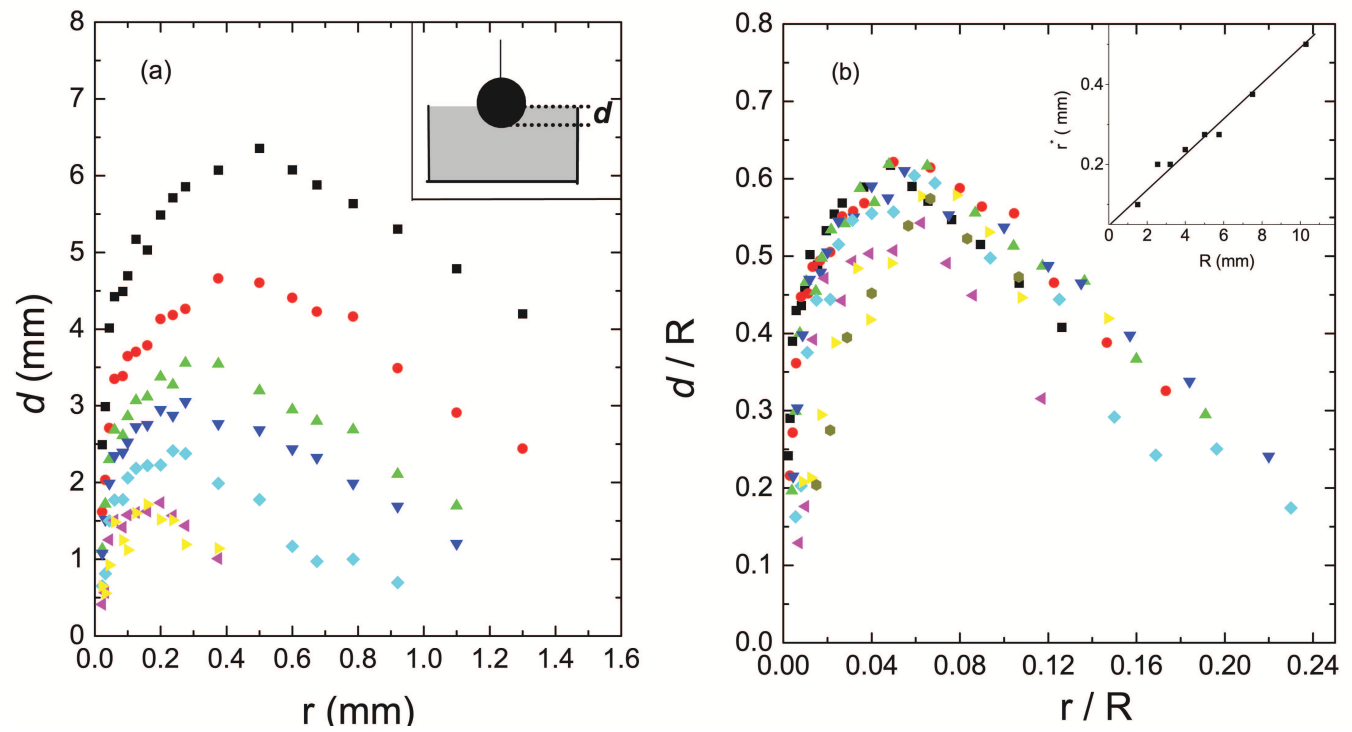

Figure 1: (a) Sinking depths of intruder balls versus bead size $r$, where $\mathrm{x}$-axis is the bead radius $r$ and y-axis is the $S D$ of the intruder ball. The curves from top to bottom correspond to $S D$ of ball radius $R=10.30,7.505 .75,5.00,4.00,3.15,2.55$ and $1.50 \mathrm{~mm}$, respectively. Inset is the schematic of the setup. (b) Curves of the ratio $d / R$ versus $r / R$, the symbols are same as in Fig. 1(a). Inset shows a linear dependence of the $r^{*}$ on $R$, where $r^{*}$ is the granule radius when the maximum SD occurs.

$d_{\max }$ is about $0.6 R$. In order to confirm $d_{\max }$ and $d$ change consistently with $r$, the $S D$ is measured as a function of $r$ at two different ball densities $10.6 \mathrm{~g} / \mathrm{cm}^{3}$ and $5.4 \mathrm{~g} / \mathrm{cm}^{3}$ and under two different packing density $\phi=0.57$ and $\phi=0.61$ as shown in Figure 2(a) and 2(b). The results show that for intruder balls of the same size $R$, the ratio of $d_{\max } / d$ is basically not a function of $r$ when changing the ball density or the bead packing density. In other words, the geometric dependent factor of $S D$ can be universal to all ball densities and granular random packings. It can also be seen from Figure 2(b) that the measured $d$ is a decreasing function of the bulk density. This 

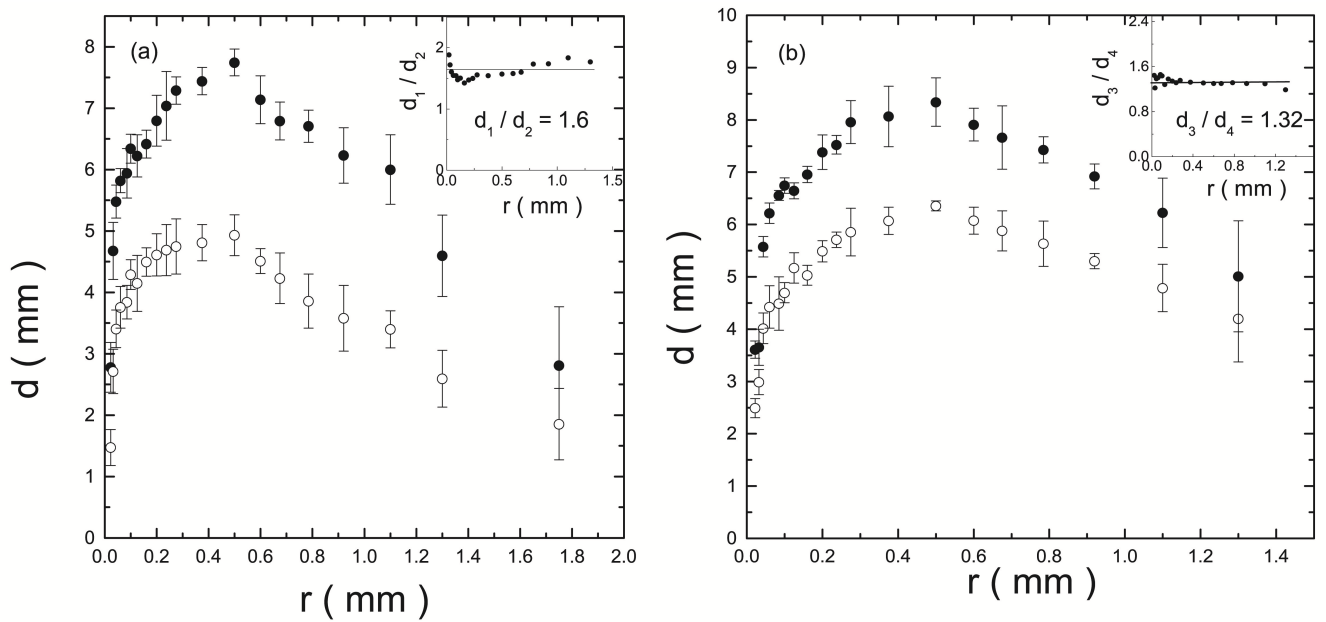

Figure 2: (a) $d-r$ dependence at intruder size $R=9.5 m m, d_{1}(r) r$ and $d_{2}(r)$ correspond to curves of ball densities $10.6 \mathrm{~g} / \mathrm{cm}^{3}$ and $5.4 \mathrm{~g} / \mathrm{cm}^{3}$, respectively. Inset is the plot of $\frac{d_{1}(r)}{d_{2}(r)}$ as a function of $r$, where $\frac{d_{1}(r)}{d_{2}(r)} \approx 1.62$. (b) $d-r$ curves of the same intruder $(\mathrm{R}=10.3 \mathrm{~mm})$ at packing density $\phi=0.57$ and $\phi=0.61$. Inset shows the ratio of $\frac{d_{3}(r)}{d_{4}(r)}$ to $r$. The average value of $\frac{d_{3}(r)}{d_{4}(r)}$ is approximately a constant i.e. 1.32

is expected, as the strength of the bed should increase with its bulk density. However, the value of $r *$ does not seem to be sensitive to the values of the bulk density. Since the general feature of Figure 2(b) is independent of the bulk densities, results reported below are all from experiments with a fixed volume fraction of $0.61 \pm 0.01$. With the same $r h o_{R}, r h o_{r}, \phi$, and using the data $d(r)$ in Fig. 1(a), we can find that the $S D$ is approximately a linear function of $R$, and thereby can be expressed as

$$
d(r R)=D(r)[R-B(r)]
$$

When $R \leq B(r), d(r R)=0 . B(r)$ is the threshold of the size of the intruder ball in a chosen granular bed of size $r$. The slope $D(r)$ and intercept $B(r)$ are plotted as a function of $r$ in Figure 3(a) and 3(b). $D(r)$ is fitted by 
115

$D(r)=a r^{\alpha}(1-b r)$, we obtain $a=1.2, b=0.4, \alpha=0.4 . B(r)$ can be fitted by $B(r)=k(r-e)$, where $k=3.2$ and $e=0.09 \mathrm{~mm}$. When $r \leq e, B(r)=0$.

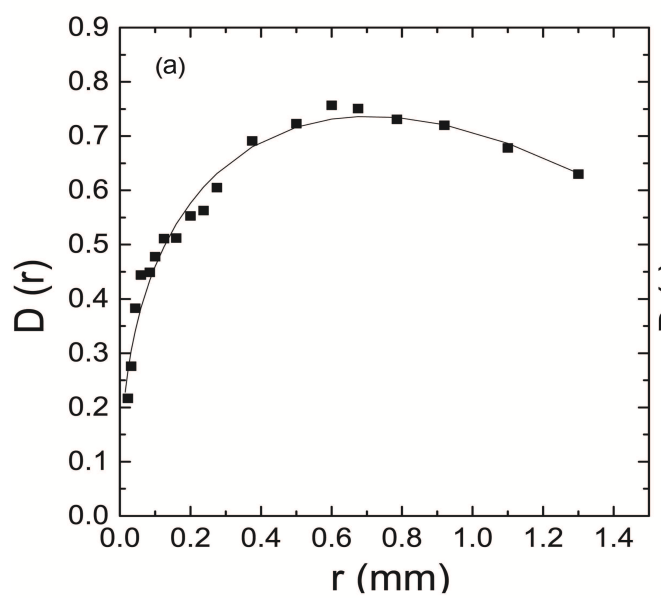

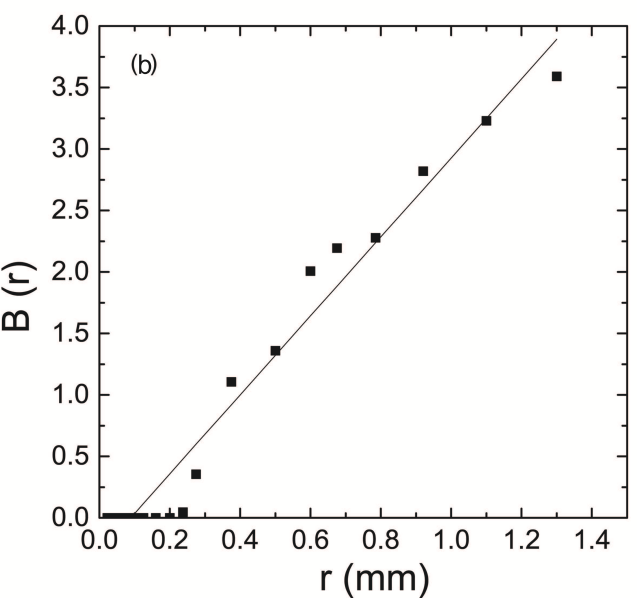

Figure 3: a) Dots are the slopes of $d(R)$. The line is a fitting of $D(r)$ by a function $r^{\alpha}(1-b r)$, where $\alpha=0.4$ and $b=0.4$. (b) Plot of intercepts $B(r)$. The $B(r)$ is fitted by a line $k(r-e)$, where $k$ and $e$ are fitting parameters, $k=3.2$ and $e=0.09 \mathrm{~mm}$.

The dependence of $S D$ on the ball density is also investigated. Filling a hollow brass shell $(R=9.5 \mathrm{~mm})$ with material of different densities, we have made balls of densities $0.9,2.6,5.4,7.3$ and $10.6 \mathrm{~g} / \mathrm{cm}^{3}$. Keeping the granular packing density $\phi=0.61$, we measure the $d_{\max }$ as a function of ball density. We find the $d_{\max }$ at $r / R \approx 0.05$. The $d_{\max }\left(\rho_{R} / \rho_{r}\right)$ curve is shown in Figure 4. It is seen that $d_{\max }$ is not a linear function of $\rho_{R} / \rho_{r}$. Fitting the curve with function $d_{\max }=\dot{A}\left(\rho_{R} / \rho_{r}-c\right)^{\beta}$, we obtain $\dot{A}=2.85, c=0.05 \beta=0.7$. Since $d_{\max }$ and $d$ follows the same dependence on ball density and volume fraction, we can write

$$
d \approx\left(\frac{\rho_{R}}{\rho_{r}}-c\right)^{\beta}
$$

$c$ is the threshold density ratio at which the ball begins to sink. When 
$\rho_{R} / \rho_{r} \leq c, d=0$. Therefore, combining (1) and (2) we obtain

$$
d=A R r^{\alpha}(1-b r)\left[1-\frac{k(r-e)}{R}\right]\left(\frac{\rho_{R}}{\rho_{r}}-c\right)^{\beta}
$$

where $A$ is a parameter related to $\phi$. We find that $A$ decreases nonlinearly with increasing $\phi$. The $\phi$ dependent of $d$ will be discussed in a separate article (our future work). In above equation when $r \leq e$ :

$$
d=A R r^{\alpha}(1-b r)\left(\frac{\rho_{R}}{\rho_{r}}-c\right)^{\beta}
$$

And when $R \leq k(r-e)$ or $\rho_{R} / \rho_{r} \leq c$, then :

$$
d=0
$$

This result is different from the fluid case. Any shearing stress can induce flow of the fluid. The submerged depth of a floating object $d$ (when $d / R$ is small) is proportional to $R\left(\rho_{s} / \rho_{l}\right)^{1 / 2}$ [14] [26]; where $\rho_{s}$ and $\rho_{l}$ are the densities of the object and the fluid, respectively. In granular media, however, $d$ is proportional to $\left(\rho_{R} / \rho_{r} c\right)^{0.75}$, and there exist thresholds $k(r e) / R$ and $c$ only above which particles yield to external forces. A small or light ball does not sink into sand. Why does the sinking depth in granules changes nonmonotonically with the size of the granules? We believe it is caused by two competing factors. One is the dependence of the number of ball-granule contact points on the sizes $r$ and $R$. With the same submerged area, the number of contacts decreases as the radius of the granules increases. The reducing number of contacts reduces the supporting the ball obtains and causes the sinking depth to increase with the increasing $r$. The other factor is the formed force chain in granules, which is larger with greater $r$ and prevent ball from sinking. When the granule size is nearly the same as 


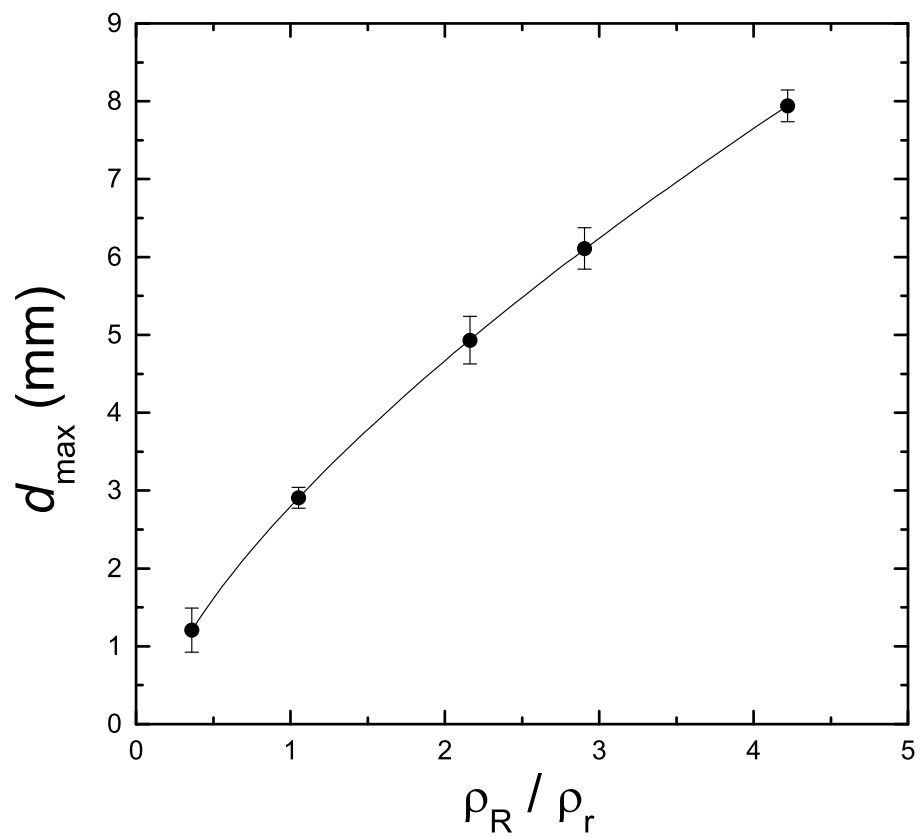

Figure 4: Plot of $d_{\max }$ versus $\rho_{R} / \rho_{r}$ with $\rho_{r}=2.5 \mathrm{~g} / \mathrm{cm}^{3}$ and $R=9.5 \mathrm{~mm}$. Fitting the data with function $d_{\max }=\hat{A}\left(\rho_{R} / \rho_{r}-c\right)^{\beta}$, we obtain $\dot{A}=2.85, c=0.05, \beta=0.7$.

the ball (assume the density of the two are the same), the sinking depth approaches zero.

\section{Conclusion}

In this work we found that the sinking depth of an intruder ball in granular media does not change monotonically with the size of the granules. There exists a maximum $d_{\max }$ at a ratio $\lambda=r / R \approx 1 / 20$, and $\lambda$ does not change with the ball mass (density) or the granular random packing. We have obtained an empirical formula of $d$ as a function of $r, R$ and $\rho_{R} / \rho_{r}$. Our results

demonstrate that the "buoyancy" of granular matter is different from that of 
solids or fluids. The sinking depth in granules does not only depend on the interaction forces, it also depends on the size of the granules. For instance, when a car or a tank moves in the desert its sinking depth depends on the sand size.

Our experimental work could not investigate the effect of coefficient of friction and the shape of grains. Such types of studies are hard to carry out experimentally; however, further research using DEM simulation is needed for better understanding of the mechanisms of this phenomenon.

\section{Acknowledgments}

The authors thank the Chinese National Science Foundation for Project No. 11172039 and University of Malaya for funding support under the research grant (BK002-2014).

\section{References}

[1] B. Andreotti, Y. Forterre, O. Pouliquen, Granular Media Between Fluid and Solid, Cambridge University Press, 2013.

[2] D. L. Blair, N. W. Mueggenburg, A. H. Marshall, H. M. Jaeger, S. R. Nagel, Force distributions in three-dimensional granular assemblies: Effects of packing order and interparticle friction, Phys. Rev. E 63 (2001) 1304.

175

[3] J. Geng, E. Longhi, R. P. Behringer, Memory in two-dimensional heap experiments, Phys. Rev. E 64 (2001) 301. 
[4] E. Kolb, J. Cviklinski, J. Lanuza, P. Claudin, , E. . ric Clement, Reorganization of a dense granular assembly: The unjamming response function, PRE 69 (2004) 031306.

180

185

195

[5] D. Slobinsky, L. A. Pugnaloni, Arch-based configurations in the volume ensemble of static granular systems, Journal of Statistical Mechanics: Theory and Experiment 2015 (2) (2015) P02005.

[6] J. F. Peters, M. Muthuswamy, J. Wibowo, A. Tordesillas, Characterization of force chains in granular material, Phys. Rev. E 72 (2005) 041307.

[7] D. Howell, R. P. Behringer, Powders and Grains, Balkema, Rotterdam, 1997.

[8] F. Radjai, Modeling force transmission in granular materials, Comptes Rendus Physique 16 (1) (2015) 3-9.

[9] E. Guyon, S. Roux, A. Hansen, D. Bideau, J.-P. Troadec, H. Crapo, Nonlocal and non-linear problems in the mechanics of disordered systems: application to granular media and rigidity problems, Rep. Prog. Phys. $53(1990) 373$.

[10] T. S. Majmudar, R. P. Behringe, Contact force measurements and stressinduced anisotropy in granular materials, Nature 435 (2005) 1079-1082.

[11] B.-L. Ge, Q.-F. Shi, R. Chand, J.-F. He, S.-P. Ma, The nature of stresses in a giant static granular column, Chinese Physics Letters 30 (3) (2013) 048101. 
[12] C. Josserand, A. V. Tkachenko, D. M. Mueth, H. M. Jaeger, Memory effects in granular materials, Phys. Rev. lett 85 (2000) 3632.

[13] C. S. O'Hern, S. A. Langer, A. J. Liu, S. R. Nagel, Random packings of frictionless particles, Phys. Rev. Lett 88 (2002) 075507.

[14] H. M. Jaeger, S. R. Nagel, R. P. Behringer, Granular solids, liquids, and gases, Rev. Mod. Phys. 68 (1996) 1259.

[15] D. I. Goldman, P. Umbanhowar, Scaling and dynamics of sphere and disk impact into granular media, Phys. Rev. E 77 (2008) 021308.

[16] K. F, V. I, L.-S. S, M. IG, Approach to failure in porous granular materials under compression., Phys. Rev. E 88 (6) (2013) 062207.

[17] S. Farhadi, A. Z. Zhu, R. P. Behringer, Stress relaxation for granular materials near jamming under cyclic compression, Phys. Rev. E 115 (2015) 188001.

[18] R. Chand, M. A. Khaskheli, A. Qadir, Y. Sandali, Q. Shi, Influence of spontaneous percolation on apparent mass at the bottom of a janssen granular column, Physica A 393 (2014) 96-100.

[19] E. Altshuler, H. Torres, A. Gonzalez-Pita, G. Sanchez-Colina, C. PerezPenichet, S. Waitukaitis, R. C. Hidalgo, Extraterrestrial sink dynamics in granular matter, arXiv 1305.6796v2.

[20] J. M. Erikson, N. W. Mueggenburg, H. M. Jaeger, S. R. Nagel, Force distribution in tree-dimensional compressible granular packs, Phys. Rev. E 66 (2002) 301. 
220

[21] J. R. de Bruyn, A. M. Walsh, Penetration of spheres into loose granular media, Can. J. Phys 82 (2004) 439-446.

[22] F. Pacheco-Vzquez, J. Ruiz-Surez, Cooperative dynamics in the penetration of a group of intruders in a granular medium, Nature Comm.

[23] F. Pacheco-Vzquez, J. C. Ruiz-Surez, Sliding through a superlight gran225

[24] F. Pacheco-Vazquez, G. A. Caballero-Robledo, J. M. Solano-Altamirano, E. Altshuler, A. J. Batista-Leyva, J. C. Ruiz-Suarez, Infinite penetration of a projectile into a granular medium, Phys. Rev. Lett 106 (2011) 218001.

230

[25] H. Katsuragi, D. J. Durian, Unified force law for granular impact cratering, Nature Physics 3 (2007) 420.

[26] I. M. Cohen, P. K. Kundu, Fluid Mechanics, 3rd Edition, Elsevier Academic Press, 2004. 